\title{
Femtosecond laser pulse propagation in a uniaxial crystal
}

\author{
DAVID HOVHANNISYAN and KOMITAS STEPANYAN \\ Epygi Laboratories AM, LLC, Yerevan, Armenia; \\ e-mails: David.Hovhannisyan@epygilab.am; \\ Komitas.Stepanyan@epygilab.am
}

(Received 2 September 2002; revision received 24 January 2003)

\begin{abstract}
The wave equation describing the vector propagation of a femtosecond laser pulse of a few optical cycles in a uniaxial crystal is solved numerically by the method of unidirectional waves. Propagation of the pulse in the direction normal to the optical axis is studied, taking into account both second- and third-order nonlinearities of the crystal. Conversion efficiency as a function of crystal length, pump intensity and pulse duration is studied. As an example, the propagation of femtosecond laser pulse of $\tau=10 \mathrm{fs}$ duration at $\lambda=810 \mathrm{~nm}$ in a $\mathrm{LiNbO}_{3}$ crystal $12 \mu \mathrm{m}$ thick is studied numerically.
\end{abstract}

\section{Introduction}

Recent progress in the generation of extremely short optical pulses has stimulated the development of propagation theory of optical pulses of a few cycles in crystals. It is known that during the propagation of such extremely short pulses in a nonlinear crystal the radiation generation takes place both at difference and at sum frequencies. At the same time the generation of difference frequencies in a medium with second-order nonlinearity is usually used for the generation of a coherent short radiation pulse in the infrared spectrum range [1].

It is clear that the approximation of slowly varying amplitude is not applicable to the description of such processes [2]. Therefore, for the description of the propagation process of a femtosecond laser pulse of a few optical cycles in an anisotropic optical crystal it is necessary to use either numerical methods or some special analytical methods.

In particular, in [3] the results of numerical simulation of propagation process of a femtosecond laser pulse with $10 \mathrm{fs}$ duration in a nonlinear potassium dihydrogen phosphate crystal with $100 \mu \mathrm{m}$ thickness obtained by the numerical integration of the Maxwell vector equation are given. There a comparison of those results with similar data obtained by the slowly varying amplitude (SVA) method is given, and it is shown that the description of the second-harmonic generation process by such short pulses by the SVA method is not correct.

So, the correct analytic description of the propagation of femtosecond laser pulse of a few optical cycles in an anisotropic nonlinear optical crystal is a very topical problem.

In [4] the analytic description of the propagation of a femtosecond laser pulse of a few optical cycles in a medium with second-order nonlinearity was given on the basis of the method of unidirectional waves (MUW). It has been shown there 
that in the approximation of small nonlinearity and negligible material dispersion the propagation of an extremely short pulse can be described adequately by the MUW.

The MUW was used also for the solution of problem of the propagation of a femtosecond laser pulse of few optical cycles in a dispersionless medium with third- and fifth-order nonlinearity [5].

In this article, the MUW for the solution of the problem of vector propagation of a pulse of a few optical cycles in a negative uniaxial crystal taking into account both second- and third-order nonlinearities is proposed.

The conversion efficiency is studied as a function of crystal length, pump intensity and pulse duration.

As an example, the propagation of a femtosecond laser pulse of $\tau=10 \mathrm{fs}$ duration at $\lambda=810 \mathrm{~nm}$ in a $\mathrm{LiNbO}_{3}$ crystal $12 \mu \mathrm{m}$ thick is studied numerically.

\section{Wave equation for the description of a non-resonant interaction between an extremely short light pulse and a uniaxial crystal}

Note that when solving the problem of vector propagation of a femtosecond laser pulse of a few optical oscillations in a uniaxial optical crystal, the usual wave separation on the first and second harmonics is not applicable.

In the general case the wave equation describing the light pulse propagation in an anisotropic nonlinear transparent medium can be written in the vector form

$$
\nabla(\nabla \cdot \mathbf{E})-\Delta \mathbf{E}+\frac{1}{c^{2}} \frac{\partial^{2} \mathbf{D}}{\partial t^{2}}=0
$$

where $\mathbf{E}=\left(E_{x}, E_{y}, E_{z}\right)$ is the electric-field intensity vector of light pulse and $\mathbf{D}=\left(D_{x}, D_{y}, D_{z}\right)$ is the electric displacement vector.

Consider a linearly polarized laser pulse $\mathbf{E}=\left(E_{x}, 0,0\right)$ of a few optical cycles with a plane wavefront propagating along the $y$ axis, normal to the optical $z$ axis of a uniaxial crystal of $3 m$ symmetry group. In this case equation (1) can be written in $x$ and $z$ components as

$$
\begin{aligned}
\frac{\partial^{2} E_{x}}{\partial y^{2}}-\frac{1}{c^{2}} \frac{\partial^{2} D_{x}}{\partial t^{2}} & =0, \\
\frac{\partial^{2} E_{z}}{\partial y^{2}}-\frac{1}{c^{2}} \frac{\partial^{2} D_{z}}{\partial t^{2}} & =0, \\
D_{y} & =0 .
\end{aligned}
$$

Thus, the electric displacement vector $\mathbf{D}$ is normal to the direction of pulse propagation. So, because $E_{y}=0$ as was mentioned above, the system of equations for the description of pulse propagation in a medium can be written as

$$
\begin{aligned}
& \frac{\partial^{2} E_{x}}{\partial y^{2}}-\frac{1 \partial^{2} E_{x}}{c^{2} \partial t^{2}}=\frac{4 \pi \partial^{2} P_{x}}{c^{2} \partial t^{2}}, \\
& \frac{\partial^{2} E_{z}}{\partial y^{2}}-\frac{1 \partial^{2} E_{z}}{c^{2} \partial t^{2}}=\frac{4 \pi \partial^{2} P_{z}}{c^{2} \partial t^{2}} .
\end{aligned}
$$


In the linear part of the medium the polarization $P_{\mathrm{L} x, \mathrm{~L} z}$, is

$$
P_{\mathrm{L} x, \mathrm{~L} z}(t)=\int_{0}^{\infty} \alpha_{x, z}(\tau) E_{x, z}(t-\tau) \mathrm{d} \tau,
$$

where $\alpha_{x, z}(t)$ is the linear susceptibility of the medium for $x, z$ polarized waves correspondingly; the electric field intensity in the integrand (4) may be expanded by the small parameter $\mu=\tau_{0 \mathrm{~T}} / T_{0}$, where $\tau_{0 \mathrm{~T}}$ is the medium response time and $T_{0}$ is the average oscillation period.

After expansion the linear polarization becomes

$$
P_{\mathrm{L} x, \mathrm{~L} z}(t)=\alpha_{0 x, 0 z} E_{x, z}(t)-\frac{\alpha_{2 x, 2 z}}{2} E_{x, z}(t),
$$

where

$$
\begin{aligned}
& \alpha_{0 x, 0 z}=\int_{0}^{\infty} \alpha_{x, z}(\tau) \mathrm{d} \tau=\alpha_{x, z}(\omega=0), \\
& \alpha_{2 x, 2 z}=-\int_{0}^{\infty} \tau^{2} \alpha_{x, z}(\tau) \mathrm{d} \tau=\alpha_{x, z}^{\prime \prime}(\omega=0),
\end{aligned}
$$

$\alpha_{0 x, 0 z}$ are the low-frequency limits of the linear susceptibilities and $\alpha_{2 x, 2 z}$ are the low-frequency limits of the second derivatives of the linear susceptibilities.

This expansion in the linear part of the medium's polarization, in particular, could be used for the description of the crystal's linear response to the laser radiation of a few optical cycles in the near-infrared spectrum range. For example, for a pulse with wavelength $\lambda_{0}=0.81 \mu \mathrm{m}\left(T_{0}=2.7 \mathrm{fs}\right)$, propagating in crystal with a linear electron response time $\tau_{0 \mathrm{~m}} \approx 0.5-1 \mathrm{fs}[6]$, the parameter $\mu=\tau_{0 \mathrm{~m}} / T_{0} \approx$ $0.18-0.36$.

In the nonlinear part of the medium's polarization of a uniaxial crystal of $3 \mathrm{~m}$ group, which is responsible for second- and third order-nonlinearities, we shall confine ourselves to the quasistatic approximation:

$$
\begin{aligned}
& P_{\mathrm{N} L x}(t)=2 d_{31} E_{x}(t) E_{z}(t)+4 \chi_{11} E_{x}^{3}(t)+12 \chi_{16} E_{z}^{2}(t) E_{x}(t), \\
& P_{\mathrm{N} L z}(t)=d_{31} E_{x}^{2}(t)+d_{33} E_{z}^{2}(t)+4 \chi_{33} E_{z}^{3}(t)+4 \chi_{31} E_{x}^{3}(t),
\end{aligned}
$$

where $d_{31}$ and $d_{33}$ are the non-zero components of the second-order crystal susceptibility tensor, and $\chi_{11}, \chi_{16}, \chi_{33}$ and $\chi_{31}$ are the non-zero components of the third-order crystal susceptibility tensor [7-9].

The quasi static approximation in the nonlinear part of the medium's polarization actually fits the assumption about the inertialess nature of the crystal nonlinear response, which takes place in the near-infrared spectrum range [6].

In the unidirectional wave approximation the system of equations (3) is reduced to the following system:

$$
\begin{aligned}
& \frac{\partial E_{x}}{\partial y}+\frac{n_{x}}{c} \frac{\partial E_{x}}{\partial t}=-\frac{2 \pi}{c n_{x}} \frac{\partial P_{1 x}}{\partial t}, \\
& \frac{\partial E_{z}}{\partial y}+\frac{n_{z}}{c} \frac{\partial E_{z}}{\partial t}=-\frac{2 \pi}{c n_{z}} \frac{\partial P_{1 z}}{\partial t},
\end{aligned}
$$


where

$$
\begin{aligned}
P_{1 x}(t) & =P_{\mathrm{L} x}(t)+P_{\mathrm{NL} x}(t), \\
P_{1 z}(t) & =P_{\mathrm{L} z}(t)+P_{\mathrm{NL} z}(t), \\
n_{x, z} & =\left(1+4 \pi \alpha_{0 x, 0 z}\right)^{1 / 2} .
\end{aligned}
$$

By substituting equations (5) and (8) into equation (9), we obtain the following equations for ordinary and extraordinary waves:

$$
\begin{gathered}
\frac{\partial E_{x}}{\partial y}+\frac{n_{x}}{c} \frac{\partial E_{x}}{\partial t}=\frac{\pi \alpha_{2 x}}{c n_{x}} \frac{\partial^{3} E_{x}}{\partial t^{3}}-\frac{4 \pi d_{31}}{c n_{x}} \frac{\partial\left(E_{x} E_{z}\right)}{\partial t}-\frac{24 \pi \chi_{11}}{c n_{x}} E_{x}^{2} \frac{\partial E_{x}}{\partial t} \\
-\frac{24 \pi \chi_{16}}{c n_{x}} \frac{\partial\left(E_{x}^{2} E_{x}\right)}{\partial t} \\
\frac{\partial E_{z}}{\partial y}+\frac{n_{z}}{c} \frac{\partial E_{z}}{\partial t}=\frac{\pi \alpha_{2 z}}{c n_{z}} \frac{\partial^{3} E_{z}}{\partial t^{3}}-\frac{4 \pi d_{31}}{c n_{z}} E_{x} \frac{\partial E_{x}}{\partial t}-\frac{4 \pi d_{33}}{c n_{z}} E_{z} \frac{\partial E_{z}}{\partial t} \\
-\frac{24 \pi \chi_{33}}{c n_{z}} E_{z}^{2} \frac{\partial E_{z}}{\partial t}-\frac{24 \pi \chi_{31}}{c n_{z}} E_{x}^{2} \frac{\partial E_{x}}{\partial t}
\end{gathered}
$$

By making substituting the dimensionless values into equation (11) via the formulae

$$
\begin{aligned}
\xi & =\frac{y n_{x}}{c \tau_{0}}, \\
\eta & =\frac{y n_{x}}{c \tau_{0}}-\frac{t}{\tau_{0}}, \\
E_{x} & =E_{0} \Phi_{x}, \\
E_{z} & =E_{0} \Phi_{z},
\end{aligned}
$$

where $2 \tau_{0}$ is the incident pulse duration, $E_{0}$ is the maximal value of the real amplitude of the field polarized along the $x$ axis at the input of the medium, and $\Phi_{x}$ and $\Phi_{z}$ are the $x$ and $z$ components respectively of the normalized value of the electrical field amplitude vector in a medium, equation (11) becomes

$$
\begin{gathered}
\frac{\partial \Phi_{x}}{\partial \xi}=-\frac{\pi \alpha_{2 x}}{n_{x}^{2} \tau_{0}^{2}} \frac{\partial^{3} \Phi_{x}}{\partial \eta^{3}}+\frac{4 \pi d_{31} E_{0}}{n_{x}^{2}} \frac{\partial\left(\Phi_{x} \Phi_{z}\right)}{\partial \eta}+\frac{24 \pi \chi_{11} E_{0}^{2}}{n_{x}^{2}} \Phi_{x}^{2} \frac{\partial \Phi_{x}}{\partial \eta} \\
+\frac{24 \pi \chi_{16} E_{0}^{2}}{n_{x}^{2}} \frac{\partial\left(\Phi_{z}^{2} \Phi_{x}\right)}{\partial \eta}, \\
\frac{\partial \Phi_{z}}{\partial \xi}+\frac{n_{x}-n_{z}}{n_{x}} \frac{\partial \Phi_{z}}{\partial \eta}=- \\
\frac{\pi \alpha_{2 z}}{n_{x} n_{z} \tau_{0}^{2}} \frac{\partial^{3} \Phi_{z}}{\partial \eta^{3}}+\frac{4 \pi d_{31} E_{0}}{n_{x} n_{z}} \Phi_{x} \frac{\partial \Phi_{x}}{\partial \eta} \frac{4 \pi d_{33} E_{0}}{n_{x} n_{z}} \Phi_{z} \frac{\partial \Phi_{z}}{\partial \eta} \\
+\frac{24 \pi \chi_{33} E_{0}^{2}}{n_{x} n_{z}} \Phi_{z}^{2} \frac{\partial \Phi_{z}}{\partial \eta}+\frac{24 \pi \chi_{31} E_{0}^{2}}{n_{x} n_{z}} \Phi_{x}^{2} \frac{\partial \Phi_{x}}{\partial \eta} .
\end{gathered}
$$


By substituting

$$
\begin{aligned}
\frac{\pi \alpha_{2 x}}{n_{x}^{2} \tau_{0}^{2}} & =b_{x}, \quad \frac{\pi \alpha_{2 z}}{n_{x} n_{z} \tau_{0}^{2}}=b_{z}, \quad \frac{4 \pi d_{31} E_{0}}{n_{x}^{2}}=a_{x z}, \\
\frac{4 \pi d_{31} E_{0}}{n_{x} n_{z}} & =a_{x x}, \quad \frac{4 \pi d_{33} E_{0}}{n_{x} n_{z}}=a_{z z}, \quad \frac{n_{x}-n_{z}}{n_{x}}=\Delta n,
\end{aligned}
$$

we find that

$$
\begin{array}{ll}
c_{x x}=\frac{24 \pi \chi_{11} E_{0}^{2}}{n_{x}^{2}}, & c_{x z}=\frac{24 \pi \chi_{16} E_{0}^{2}}{n_{x}^{2}}, \\
e_{x x}=\frac{24 \pi \chi_{31} E_{0}^{2}}{n_{x} n_{z}}, & e_{z z}=\frac{24 \pi \chi_{33} E_{0}^{2}}{n_{x} n_{z}} .
\end{array}
$$

The final equations will be

$$
\begin{gathered}
\frac{\partial \Phi_{x}}{\partial \xi}=-b_{x} \frac{\partial^{3} \Phi_{x}}{\partial \eta^{3}}+a_{x z} \frac{\partial\left(\Phi_{x} \Phi_{z}\right)}{\partial \eta}+c_{x x} \Phi_{x}^{2} \frac{\partial \Phi_{x}}{\partial \eta}+c_{x z} \frac{\partial\left(\Phi_{z}^{2} \Phi_{x}\right)}{\partial \eta} \\
\frac{\partial \Phi_{z}}{\partial \xi}+\Delta n \frac{\partial \Phi_{z}}{\partial \eta}=-b_{z} \frac{\partial^{3} \Phi_{z}}{\partial \eta^{3}}+a_{x x} \Phi_{x} \frac{\partial \Phi_{x}}{\partial \eta}+a_{z z} \Phi_{z} \frac{\partial \Phi_{z}}{\partial \eta} \\
+e_{z z} \Phi_{z}^{2} \frac{\partial \Phi_{z}}{\partial \eta}+e_{x x} \Phi_{x}^{2} \frac{\partial \Phi_{x}}{\partial \eta}
\end{gathered}
$$

The system of equations (15) describing the propagation of femtosecond laser pulse of a few optical cycles in a negative uniaxial crystal in the direction normal to optical axis taking into account both the second- and third-order nonlinearities does not have an analytic solution.

The first terms on the right-hand sides of the system of equations (15) describe a linear dispersed broadening of femtosecond pulses of ordinary and extraordinary polarizations. Here the lengths of dispersed broadening for the pulses of ordinary and extraordinary polarizations are determined in the following way:

$$
\begin{aligned}
& L_{\mathrm{D} x}=\frac{c n_{x} \tau_{0}^{3}}{\pi \alpha_{2 x}}=\frac{c \tau_{0}}{b_{x} n_{x}}, \\
& L_{\mathrm{D} z}=\frac{c n_{z} \tau_{0}^{3}}{\pi \alpha_{2 z}}=\frac{c \tau_{0}}{b_{z} n_{x}} .
\end{aligned}
$$

The remaining terms on the right-hand sides of the system of equations (15) describe the processes of nonlinear interaction of pulses of ordinary and extraordinary polarizations, caused by both second and third-order nonlinearities of the nonlinear crystal. Here the nonlinear lengths, caused by second- and thirdorder nonlinearities of nonlinear crystal, are determined as

$$
\begin{aligned}
& L_{\mathrm{NL} x z}^{(2)}=\frac{c \tau_{0}}{n_{x} a_{x z}}, \quad L_{\mathrm{NL} x x}^{(2)}=\frac{c \tau_{0}}{n_{x} a_{x x}}, \quad L_{\mathrm{NL} z z}^{(2)}=\frac{c \tau_{0}}{n_{x} a_{z z}}, \\
& L_{\mathrm{NL} x x}^{(3)}=\frac{c \tau_{0}}{n_{x} c_{x x}}, \quad L_{\mathrm{NL} x z}^{(3)}=\frac{c \tau_{0}}{n_{x} c_{x z}}, \quad L_{\mathrm{NL} z z}^{(3)}=\frac{c \tau_{0}}{n_{x} e_{z z}}, \quad L_{\mathrm{NL} x x}^{(3)^{1}}=\frac{c \tau_{0}}{n_{x} e_{x x}} .
\end{aligned}
$$


The second summand on the left-hand side of the second equation of system (15), proportional to $\Delta n$, describes the inequality of the pulse velocities of ordinary and extraordinary polarizations in a crystal.

Detuning of velocities causes a bias of the pulse of extraordinary polarization during propagation in a nonlinear crystal in relation to the pulse of an ordinary wave. This causes the decrease in the radiation conversion efficiency. As a characteristic parameter the following length can be used:

$$
L_{\mathrm{u}}=\frac{c \tau_{0}}{n_{x}-n_{z}}
$$

The effect of detuning of the velocities on the efficiency of harmonic generation depends on the ratio of the interaction length $z$ to the length $L_{\mathrm{u}}$. When $z<L_{\mathrm{u}}$, stationary harmonic generation takes place. When $z>L_{\mathrm{u}}$, the harmonic oscillation mode is essentially unsteady.

\section{Results of numerical simulations}

Figures 1 and 2 show the results of numerical simulations of the system of equations (15), describing the propagation of femtosecond pulses of ordinary polarization $\left(\Phi_{x}(\xi, \eta)\right)$ and extraordinary polarization $\left(\Phi_{z}(\xi, \eta)\right)$ in a uniaxial optical crystal $\mathrm{LiNbO}_{3}$, with the following boundary conditions for pulse fields of ordinary and extraordinary waves at the input of the crystal:

$$
\begin{aligned}
& \Phi_{x}(0, \eta)=\exp \left(-\frac{\eta^{2}}{2}\right) \cos (W \eta), \\
& \Phi_{z}(0, \eta)=0
\end{aligned}
$$

where $W=\omega_{0} \tau_{0}$ and $\omega_{0}$ is the spectrum central frequency of the input videopulse.

Computations are made for the following values: the input pulse duration was $2 \tau_{0}=10 \mathrm{fs}$, the central wavelength $\lambda_{0}=2 \pi c / \omega_{0}=0.81 \mu \mathrm{m}$, the incident radiation intensity $I=5 \times 10^{8} \mathrm{~W} \mathrm{~cm}^{2}, n_{x}=2.159, n_{z}=2.093, d_{31}=-5.8 \times 10^{-12} \mathrm{~m} \mathrm{~V}^{-1}, d_{33}=$ $-3.4 \times 10^{-11} \mathrm{~m} \mathrm{~V}^{-1}, \quad a_{x x}=-0.99 \times 10^{-3}, \quad a_{x z}=-0.96 \times 10^{-3}, \quad a_{z z}=-5.81 \times 10^{-3}$, $b_{x}=1.1481 \times 10^{-4}, b_{z}=4.7160 \times 10^{-5}, c_{x x}=7.9221 \times 10^{-6}, c_{x z}=2.3133 \times 10^{-6}, e_{z z}=$ $8.1719 \times 10^{-6}, \mathrm{e}_{x x}=2.4516 \times 10^{-5}$ and the crystal length $z=12 \mu \mathrm{m}$. For predetermined numerical values of the dispersed broadening lengths for pulses of ordinary and extraordinary polarization, $L_{\mathrm{D} x}=6 \mathrm{~mm}$ and $L_{\mathrm{D} z}=14,7 \mathrm{~mm}$, the nonlinear lengths determined by second- and third-order nonlinearities of a crystal are $L_{\mathrm{NL} x z}^{(2)}=0.724 \mathrm{~mm}, L_{\mathrm{NL} x x}^{(2)}=0.702 \mathrm{~mm}, L_{\mathrm{NL} z z}^{(2)}=0.12 \mathrm{~mm}, L_{\mathrm{NL} x x}^{(3)}=87.7 \mathrm{~cm}, L_{\mathrm{NL} x z}^{(3)}=$ $30.03 \mathrm{~m}, L_{\mathrm{NL} z z}^{(3)}=6.95 \mathrm{~m}, L_{\mathrm{NL} x x}^{(3)^{\prime}}=2.32 \mathrm{~m}$, and the length $L_{\mathrm{u}}=45.45 \mu \mathrm{m}$. As evident from aforementioned numerical values, the length of our selected crystal $z<L_{\mathrm{u}}$. This means that in this particular case the stationary harmonic oscillation mode occurs.

In figure $1(a)$ the time dependence is shown for a femtosecond pulse field with ordinary polarization at the crystal input $(z=0)$; in figure $1(b)$ the time dependence is shown for a femtosecond pulse field with ordinary polarization at the crystal output; in figure $1(c)$ the time dependence is shown for a femtosecond pulse field with extraordinary polarization at the crystal output.

In figure $2(a)$ the normalized amplitude spectrum for a femtosecond pulse field with ordinary polarization $\left(S_{x}(\omega) / S_{x 0}(\omega)\right)$ at the crystal input is given; 

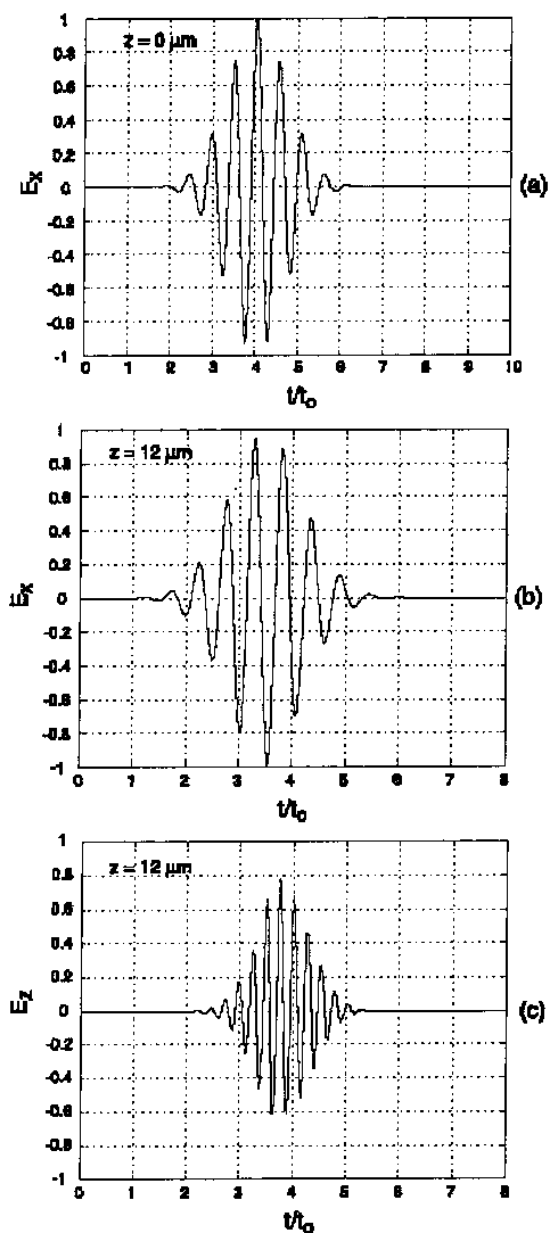

Figure 1. Dynamics of a femtosecond pulse with an ordinary polarization with $10 \mathrm{fs}$ duration $\left(\lambda_{0}=0.81 \mu \mathrm{m}\right)$ and intensity $5 \times 10^{8} \mathrm{~W} \mathrm{~cm}^{-2}$, propagated in a $\mathrm{LiNbO}_{3}$ crystal, when $(a) z=0 \mu \mathrm{m}$ and $(b) z=12 \mu \mathrm{m}$. (c) Femtosecond pulse with an extraordinary polarization at the crystal's output.

in figure $2(b)$ the normalized amplitude spectra for femtosecond pulse fields with ordinary polarization $\left(S_{x}(\omega) / S_{x 0}(\omega)\right)$ and extraordinary polarization $\left(S_{z}(\omega) / S_{x 0}(\omega)\right)$ at the crystal output are given.

According to the numerical calculations results and as shown in figure 2, the radiation conversion ratio $\gamma \sim\left[S_{z}(\omega) / S_{x 0}(\omega)\right]^{2}$ at the double frequency $2 \omega_{0}$, for the aforementioned numerical values of parameters, is approximately $22 \%$ and, at frequencies near zero frequency and $3 \omega_{0}, \gamma<0.18 \%$. It is obvious that only those spectral components that are in the passband of a crystal will propagate in a medium. In particular, for our $\mathrm{LiNbO}_{3}$ crystal the passband is $0.33-5.5 \mu \mathrm{m}$, $3.4272 \times 10^{14}-5.712 \times 10^{15} \mathrm{rad} \mathrm{s}^{-1}$ [9].

Figure 3 shows the dependence of the radiation conversion ratio $\gamma \sim\left[S_{z}(\omega) /\right.$ $\left.S_{x 0}(\omega)\right]^{2}$ at frequencies near the double frequency $2 \omega_{0}$ from the crystal length for the femtosecond pulse durations $\tau_{0}=5$ and $10 \mathrm{fs}$ and for an incident radiation intensity $I=5 \times 10^{8} \mathrm{~W} \mathrm{~cm}^{-2}$. 

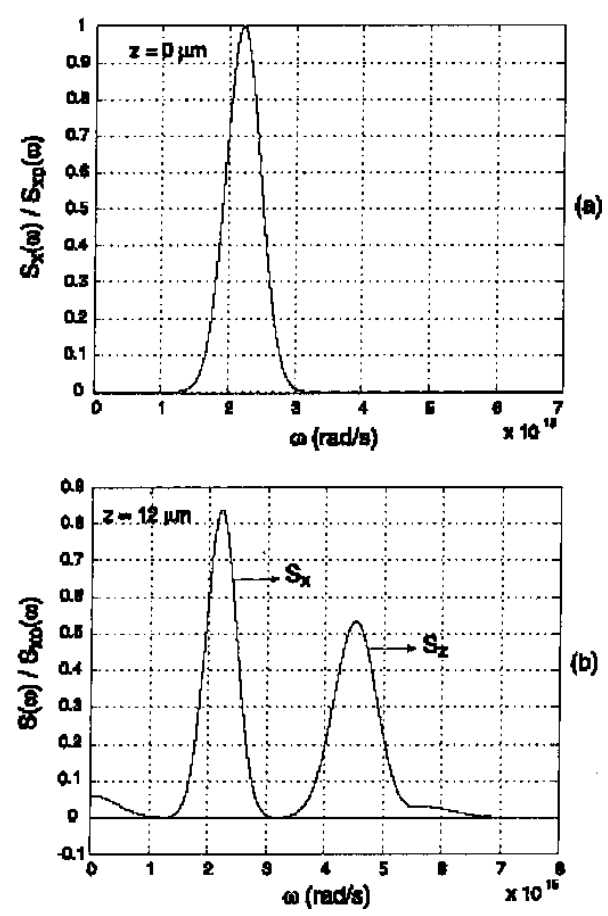

Figure 2. (a) Normalized amplitude spectrum for a femtosecond pulse field with an ordinary polarization $\left(S_{x}(\omega) / S_{x 0}(\omega)\right)$ at the input of the crystal. (b) Normalized amplitude spectra for femtosecond pulse fields with ordinary polarization $\left(S_{x}(\omega) /\right.$ $\left.S_{x 0}(\omega)\right)$ and extraordinary polarization $\left(S_{z}(\omega) / S_{x 0}(\omega)\right)$ at the output of the crystal.

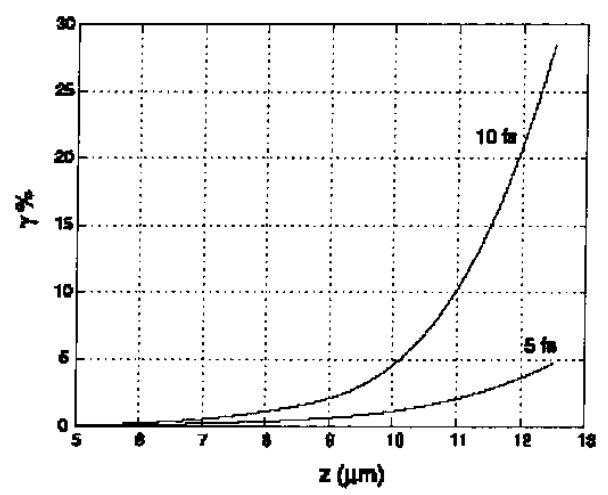

Figure 3. Dependence of the radiation conversion ratio $\gamma \sim\left[S_{z}(\omega) / S_{x 0}(\omega)\right]^{2}$ at frequencies near $2 \omega_{0}$ from the length of crystal, for femtosecond pulse durations $\tau_{0}=5$ and $10 \mathrm{fs}$ and incident radiation intensity $I=5 \times 10^{8} \mathrm{~W} \mathrm{~cm}^{-2}$.

As shown in the figure, for a given crystal length, as the femtosecond pulse duration decreases, the efficiency of conversion to the second harmonic also decreases. From the physical point of view, this means that, as the pulse duration decreases, that is as the pulse spectrum width increases, the phase synchronism condition holds for a minority of the pulse spectrum. This, in turn, causes the decrease in the radiation conversion efficiency at frequencies near the double frequency. 


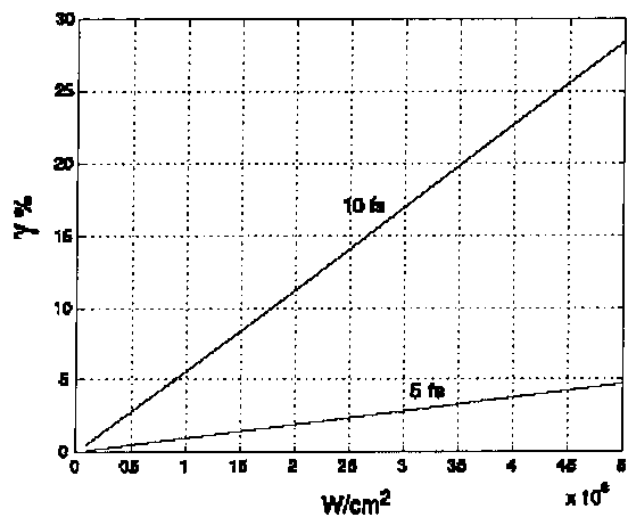

Figure 4. Dependence of the radiation conversion ratio $\gamma \sim\left[S_{z}(\omega) / S_{x 0}(\omega)\right]^{2}$ at frequencies near $2 \omega_{0}$ from the incident radiation intensity, for femtosecond pulse durations $\tau_{0}=5$ and $10 \mathrm{fs}$ and crystal length $z=12 \mu \mathrm{m}$.

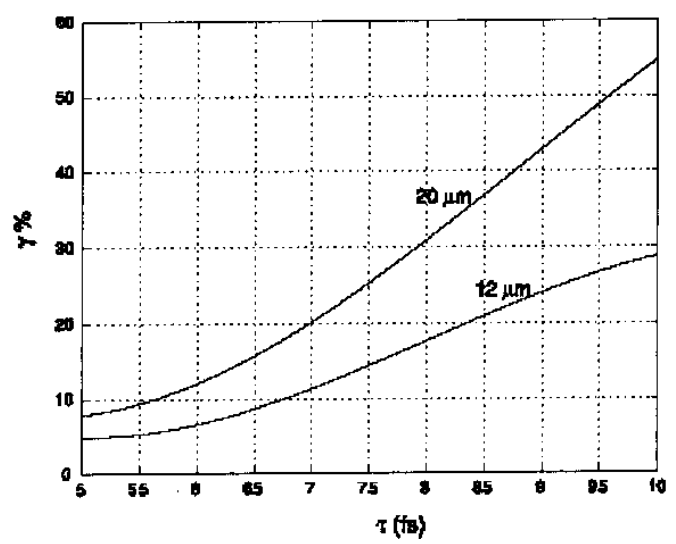

Figure 5. Dependence of the radiation conversion ratio $\gamma \sim\left[S_{z}(\omega) / S_{x 0}(\omega)\right]^{2}$ at frequencies near $2 \omega_{0}$ from the femtosecond pulse duration, for incident radiation intensity $I=5 \times 10^{8} \mathrm{~W} \mathrm{~cm}^{-2}$ and crystal lengths $z=12$ and $20 \mu \mathrm{m}$.

Figure 4 shows the dependence of the radiation conversion ratio $\gamma \sim\left[S_{z}(\omega) /\right.$ $\left.S_{x 0}(\omega)\right)^{2}$ at frequencies near the double frequency $2 \omega_{0}$ from the incident radiation intensity for the femtosecond pulse durations $\tau_{0}=5$ and $10 \mathrm{fs}$ and for a crystal length $z=12 \mu \mathrm{m}$.

Figure 5 shows the dependence of the radiation conversion ratio $\gamma \sim\left[S_{z}(\omega) /\right.$ $\left.S_{x 0}(\omega)\right]^{2}$ at frequencies near the double frequency $2 \omega_{0}$ from the femtosecond pulse duration for an incident radiation intensity $I=5 \times 10^{8} \mathrm{~W} \mathrm{~cm}^{-2}$ and crystal lengths $z=12$ and $20 \mu \mathrm{m}$.

As follows from the numerical calculations, in contrast with [4], during femtosecond pulse propagation in a nonlinear crystal, taking into account thirdorder nonlinearity, the generation of sum frequencies concentrated near the triple frequency of the original pulse also takes place.

According to the numerical calculations of the system of equations (15), during the propagation of femtosecond laser pulse of a few optical oscillations in a negative uniaxial crystal in the direction normal to optical axis, taking into account both second- and third-order nonlinearities, in case when phase synchronism is 
not applicable to the whole broad pulse spectrum, the pulse of extraordinary waves arises and is enhanced in the crystal, and in its spectrum the sum and difference frequencies regions are clearly defined. The frequencies corresponding to the maximum values in the sum frequencies region do not equal $2 \omega_{0}$ and $3 \omega_{0}$ of the original pulse. They can be more or less than $2 \omega_{0}$ and $3 \omega_{0}$, depending on the direction from the central frequency of original pulse $\omega_{0}$ in which the frequency components, not subject to the phase synchronism condition for second- and third-harmonic generation, are biased. The maximum in the difference frequencies region always occurs at zero frequency. The existence of constant component of the field is noticeable also in the time profile of extraordinary pulse. The broad spectrum of the extraordinary pulse determines the original pulse duration.

\section{Conclusions}

In this paper we derived a system of nonlinear wave equations (15) that describes the propagation of a femtosecond laser pulse of a few optical oscillations in a negative uniaxial crystal in the direction normal to the optical axis, taking into account both second- and third-order nonlinearities, in the case when phase synchronism is not applicable to the whole broad pulse spectrum. In the linear part of the medium's polarization an expansion in the small parameter, equal to the ratio of the medium's response time to the average oscillation period of the femtosecond pulse, is made. This expansion could be used for to decribe the uniaxial crystal's linear response to the laser radiation of a few optical oscillations in the near-infrared spectrum range. In the nonlinear part of the polarization of a uniaxial crystal of the $3 m$ group, responsible for the second- and third-order nonlinearities, we confine ourselves to considering the quasistatic approximation that takes place in the nearinfrared spectrum range and for small thicknesses of the nonlinear crystal.

From the numerical analysis of system (15) it follows that consideration of third-order nonlinearity causes in the optical femtosecond pulse spectrum the spectral components of an extraordinary polarization concentrated in the triplefrequency region of original pulse.

In this paper the dependences of the radiation conversion ratio $\gamma \sim\left[S_{z}(\omega) /\right.$ $\left.S_{x 0}(\omega)\right]^{2}$ are obtained, both from the femtosecond pulse duration and intensity, and from the crystal length at frequencies near the double frequency $2 \omega_{0}$.

It is shown that the conversion efficiency in the vicinity of the double frequency $2 \omega_{0}$ for pulse with a duration of $10 \mathrm{fs}$ is equal to about $22 \%$, while for a pulse of $5 \mathrm{fs}$ duration it has a value of about $3.7 \%$.

\section{Acknowledgments}

The authors would like to acknowledge Zaven Kalayjian and Suren Soghomonyan for their editorial and technical feedback, as well as Epygi Laboratories AM for supporting this work.

\section{References}

[1] Kaindl, R. A., Smith, D. C., Joschko, M., Hasselbeck, M. P., Woerner, M., and Elsaesser, T., 1998, Optics Lett., 23, 861.

[2] Sidick, E., Knoesen, A., and Diens, A., 1995, J. opt. Soc. Am. B, 12, 1704.

[3] Bourgeade, A., and Freysz, E., 2000, J. opt. Soc. Am. B, 17, 226. 
[4] Akopyan, A. A., and Hovhannisyan, D. L., 1998, Quant. Electron., 25, 954 (in Russian).

[5] Hovhannisyan, D. L., 2001, Optics Commun., 196, 103.

[6] Boyd, R. W., 1991, Nonlinear Optics (New York: Academic Press).

[7] Banks, P. S., Feit, M. D., and Perry, M. D., 2002, J. opt. Soc. Am. B, 19, 102.

[8] Yang, X., and XIE, S. 1995, Appl. Optics, 34, 6130.

[9] Gurzadyan, G. G., Dmitriev, W. G., and Nikoghosyan, D. N., 1991, Nonlinear Optical Crystals: Properties and Applications in Quantum Electronics (Moscow: Radio and Communication). 